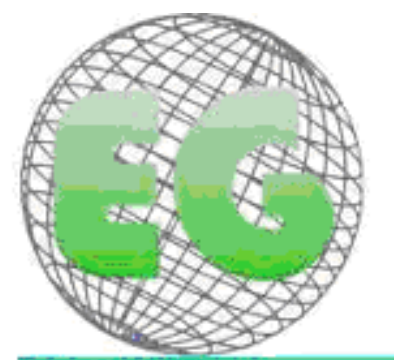

15SN 1695-6) is:

\title{
CONOCIMIENTO DE ENFERMERÍA ACERCA DE LOS MÉTODOS ANTICONCEPTIVOS EN EL CONTEXTO DEL PROGRAMA DE SALUD DE LA FAMILIA
}

CONHECIMENTO DE ENFERMEIROS SOBRE MÉTODOS CONTRACEPTIVOS NO CONTEXTO DO PROGRAMA SAÚDE DA FAMÍLIA

\section{${ }^{*}$ Moura Lopes, E, ${ }^{* *}$ Ferreira da Silva, S, ${ }^{* *}$ Costa de Moraes, ML, ${ }^{* * *}$ De Sousa Aquino, $\mathrm{P},{ }^{* * * *}$ Américo, CF, ${ }^{* * * * *}$ Bezerra Pinheiro, AK.}

\begin{abstract}
*Mestranda em Enfermagem. Universidade Federal do Ceará. ** Acadêmica de Enfermagem. Universidade Federal do Acre. ${ }^{* * *}$ Mestre em Enfermagem. Doutoranda em Enfermagem. Universidade Federal do Ceará. ${ }^{* * * *}$ Enfermeira. Universidade Federal do Ceará. ${ }^{* * * * *}$ Doutora em Enfermagem. Professora Adjunto do Departamento de Enfermagem. Universidade Federal do Ceará. Brasil..
\end{abstract}

\section{Palabras chave: conhecimento, enfermagem, anticoncepção \\ Palabras clave: conocimiento, enfermería, anticoncepción}

Keywords: Knowledge, Nursing, and Contraception

\section{RESUMEN}

La anticoncepción ha ganado más espacio para el debate en los últimos 40 años. La enfermera como profesional que tiene participación activa en este proceso, debe ayudar, efectivamente, a las personas que optan por buscar la anticoncepción y el servicio de salud pública. El objetivo fue evaluar los conocimientos de las enfermeras acerca de los métodos anticonceptivos en el contexto del Programa de Salud de la Familia (PSF) acerca de los métodos anticonceptivos. Estudio descriptivo con enfoque cuantitativo, realizado entre julio y noviembre de 2007. Los participantes del estudio fueron 71 enfermeras del PSF de Fortaleza. La mayoría de las enfermeras, 59 (83,1\%), es de sexo femenino y 22 (31\%) trabajan en el PSF alrededor de 4 a 6 años. Las enfermeras demostraron un mayor conocimiento acerca de los métodos naturales de anticoncepción y los items con mayor porcentaje de errores fueron los métodos de barrera. Se encontró que el conocimiento de las enfermeras acerca de métodos anticonceptivos no era suficiente, necesitando más actualización y capacitación de estos profesionales. 


\section{RESUMO}

A contracepção vem ganhando mais espaços de discussão nos últimos 40 anos. O enfermeiro como profissional que tem participação ativa neste processo, deve auxiliar, efetivamente, as pessoas que optam pela contracepção e buscam o serviço público de saúde. Objetivou-se avaliar o conhecimento de enfermeiros sobre métodos contraceptivos no contexto do Programa Saúde da Família (PSF) acerca dos métodos contraceptivos. Estudo descritivo com abordagem quantitativa, realizado entre julho e novembro de 2007. Participaram do estudo 71 enfermeiros do PSF de Fortaleza. A maioria dos enfermeiros, $59(83,1 \%)$, é do sexo feminino e $22(31 \%)$ trabalham no PSF há cerca de 4 a 6 anos. Os enfermeiros demonstraram maior conhecimento acerca dos métodos contraceptivos naturais e os itens com maiores percentuais de erros foram os métodos de barreira. Verificou-se que o conhecimento dos enfermeiros sobre os métodos contraceptivos não foi adequado, necessitando de maior atualização e capacitação desses profissionais.

\section{ABSTRACT}

Contraception has gained room for debate in the last 40 years. The nurse as a professional that has active participation in this process to help. In practice, some people are opting to look for contraception and service in public health care. The objective was to evaluate the nurse's knowledge of contraceptive methods in the context of the Family Health Program (FHP) regarding contraceptive methods. Descriptive study with a quantitative focus, carried out between July and November 2007. The participants of the study were 71 nurses. Most of nurses, 59 (83.1\%), were females and $22(31 \%)$ worked there for about 4 to 6 years. Nurses showed a higher knowledge regarding natural contraceptive methods and the topics with the highest percentage of errors were barrier methods. It was found that the nurses' knowledge was not enough regarding contraceptive methods and therefore they need more training and updating for these professionals.

\section{INTRODUCCIÓN}

La preocupación con el número de hijos y cuándo tenerlos es un asunto cada vez más en foco. Cuestiones como la inserción y promoción de la mujer en el mercado laboral, problemas financieros y sociales y la participación masculina en el planeamiento familiar hacen a las mujeres y a las parejas posponer la maternidad y la paternidad y optar por métodos anticonceptivos.

El planeamiento familiar se compone de acciones de control de la fecundidad, garantizando la igualdad de derechos para hombres y mujeres en relación a la prole ${ }^{1}$. Por lo tanto, permite la elección de la pareja acerca del mejor momento para la concepción e implica el conocimiento acerca de la eficacia, las condiciones, indicaciones y contraindicaciones de los métodos anticonceptivos existentes.

La libertad de elección del método anticonceptivo debe ser un derecho otorgado a la pareja y comprende el acceso a la información sobre su uso y sus características. Por lo tanto, necesita de información profesional para que la elección ocurra de la forma más saludable, manteniendo la autonomía del cliente.

En relación a la salud de la mujer, el marco inicial que consideró su complejidad y la atención integral fue el surgimiento del Programa Integrado de Salud de la Mujer (PAISM) en 1984. EI PAISM se produjo en respuesta a las presiones para la instalación de un programa de control de la fecundidad en Brasil y trasladó a los límites de las funciones reproductivas ${ }^{2}$.

En 2003 fue preparado por el servicio técnico de salud de la mujer, el Programa Nacional de Atención Integral a la Salud de la Mujer (PANAISM), que refleja el compromiso del Gobierno Federal de Brasil en la aplicación de políticas públicas para garantizar los derechos de la 
mujer. Una de las áreas de trabajo es la atención para el planeamiento familiar, cuyo objetivo es mejorar la información y el acceso a los métodos anticonceptivos ${ }^{3}$.

Como estrategia de atención integral a la salud de los clientes, se puso en marcha en 1994, el Programa de Salud de la Familia (PSF), que representa a la atención primaria a la salud (nivel municipal) y es un conjunto de acciones centradas en un enfoque preventivo. Esta es una propuesta de trabajo en equipos formados por médicos, enfermeros, auxiliares de enfermería y trabajadores de la comunidad, que tienen responsabilidad para la salud de cerca de 1000 famílias $^{4}$.

Una de las acciones básicas del PSF en el programa de atención de la salud reproductiva es la orientación para el planeamiento familiar. Esto se define como un conjunto de acciones que proporciona los recursos científicamente probados para concepción o anticoncepción. Tratase de un derecho sexual y reproductivo que debe ser garantizado por el Gobierno ${ }^{5}$.

Entre los componentes del equipo de salud de la familia, el personal de enfermería se muestra como un elemento clave en la aplicación, ejecución y continuidad de las prácticas de planeamiento familiar, siendo fundamental la capacidad y la competencia por parte de este profesional. La actualización y capacitación son necesarias para garantizar que el profesional sea capaz de dar informaciones precisas y responder a las preguntas de los usuarios, contribuyendo a la autonomía de sus clientes. Se necesita conocimiento técnico, científico y cultural, con el fin de atender a la necesidad de servicios de salud sexual y reproductiva de los clientes, incluyendo la capacidad de proporcionar información y comunicarse adecuadamente ${ }^{4}$. Es necesario que los profesionales de salud conozcan las necesidades de sus clientes para prestar asistencia en su totalidad, promoviendo la salud y generando la calidad de vida ${ }^{6}$.

Dada la importancia del enfermero en la atención a los clientes que buscan el servicio público de planeamiento familiar, en particular los métodos de anticoncepción, es necesario saber el conocimiento de los enfermeros que tratan directamente con estas personas.

Así, el objetivo fue verificar los conocimientos de los enfermeros sobre los métodos anticonceptivos en el contexto del Programa de Salud de la Familia.

\section{METODOLOGÍA}

Tratase de un estudio descriptivo, transversal y con abordaje cuantitativo, realizado en Centros de Salud de la Familia de Fortaleza (CSF). La administración municipal de esta ciudad se subdivide en seis Secretarías Ejecutivas Regionales (SER). Para este estudio, hemos elegido las SER I y III, que fueron deliberadamente seleccionadas por un mayor acceso por parte de los investigadores, siendo más fácil la realización del estudio.

La población del estudio estuvo formada por todos los enfermeros que trabajan en el Programa de Salud de la Familia de las SER I y III, siendo un total de 88 profesionales. La muestra consistió de 71 enfermeros. Se utilizaron como criterios de inclusión ser enfermero que trabaja en el PSF de las SER I y III, y aceptar participar del estudio. También se excluyeron los profesionales que estaban de vacaciones o licencia por maternidad 0 enfermedad durante la recolección de datos.

La recopilación de datos se llevó a cabo entre agosto y noviembre de 2007. Se aplicó un cuestionario estructurado que contiene 34 preguntas objetivas, con elementos de verdadero o falso sobre los métodos anticonceptivos, y las preguntas subjetivas, que tratan las 
informaciones de identificación profesional. Las preguntas específicas sobre los métodos anticonceptivos fueron extraídas de manuales técnicos, que fueron utilizados como parámetros para definir aciertos o errores.

Los datos fueron introducidos y organizados en el programa Excel, utilizando tablas. Se aplicó el programa Statistical Package for Social Sciences 11.0 (SPSS) para almacenar y codificar los datos. Se realizó el análisis estadístico descriptivo y la discusión con la literatura adecuada.

El estudio fue presentado al Comité de Ética en Investigación de la Universidad Federal de Ceará y aprobado de acuerdo con el protocolo 180/07. El estudio ha seguido las directrices y normas para la investigación con seres humanos de la Resolución 196/96 del Consejo Nacional de Salud, Ministerio de Salud, en virtud del cual todos los participantes firmaron un consentimiento después de ser informado acerca de los objetivos del estudio.

\section{RESULTADOS Y ANÁLISIS}

Para una caracterización general de la muestra se investigó el sexo, el tiempo total de trabajo y el tiempo de trabajo en el PSF de los enfermeros.

[Tabla 1 - Distribución del los enfermeros por sexo, tiempo de trabajo total y en PSF. Fortaleza, 2007]

TABELA I. Distribuição dos enfemeiros segundo sexo, tempo total de trabalho e em PSF. Fortaleza, 2007.

\begin{tabular}{lll}
\hline $\begin{array}{l}\text { Variáveis }(\mathbf{n}=7 \mathbf{1}) \\
\text { Sexo }\end{array}$ & $\mathbf{n}$ & $\mathbf{\%}$ \\
$\quad$ Masculino & 12 & 16,9 \\
$\quad$ Feminino & 59 & 83,1 \\
Tempo total de trabalho em anos & & \\
$0-3$ & 12 & 17 \\
$4-6$ & 22 & 31,0 \\
$7-9$ & 23 & 32,3 \\
$10-12$ & 9 & 12,7 \\
$>12$ & 5 & 7 \\
Tempo de trabalho em PSF em anos & & \\
$0-3$ & 18 & 25,4 \\
$4-6$ & 20 & 28,2 \\
$7-9$ & 22 & 31 \\
$10-12$ & 10 & 14,1 \\
$>12$ & 1 & 1,4 \\
\hline
\end{tabular}

La población de estudio fue predominantemente femenina, con un total de 59 (83,1\%) profesionales. Los hombres representaron 12 profesionales (16,9\%) enfermeros. 
La mayoría de los profesionales, 23 (32,3\%), tiene entre 7 y 9 años de trabajo, seguidos de los que tienen entre 4 y 6 años en la profesión, 22 (31\%). Sobre el tiempo de trabajo en el PSF, 22 (31\%) profesionales tienen entre 7 y 9 años y $20(28,2 \%)$ tienen entre 4 y 6 años. Para los cursos de especialización, de los 70 profesionales que respondieron a este tema, $64(91,4 \%)$ reportaron haber al menos un curso de especialización.

A los enfermeros se les preguntó sobre los métodos anticonceptivos en temas específicos.

[Tabla 2 - Distribución del número de aciertos y errores acerca de los métodos de anticoncepción de los enfermeros del PSF. Fortaleza, 2007]

Tabela II- Distribuição do número de acertos e erros acerca de métodos contraceptivos de enfermeiros do PSF. Fortaleza, 2007

\begin{tabular}{|c|c|c|c|c|c|c|}
\hline \multirow[t]{2}{*}{ Tema } & \multicolumn{2}{|c|}{ Acertos } & \multicolumn{2}{|c|}{ Erros } & \multicolumn{2}{|c|}{ Total } \\
\hline & $\mathbf{n}$ & $\%$ & $\mathbf{n}$ & $\%$ & $\mathbf{n}$ & $\%$ \\
\hline \multicolumn{7}{|l|}{ Métodos hormonais } \\
\hline $\begin{array}{l}\text { Escolha do contraceptivo oral combinado para } \\
\text { iniciar o uso }\end{array}$ & 58 & 81,7 & 13 & 18,3 & 71 & 100 \\
\hline Período de iniciação do contraceptivo & 31 & 43,7 & 40 & 56,3 & 71 & 100 \\
\hline Uso da injeção trimestral durante a amamentação & 42 & 59,2 & 29 & 40,8 & 71 & 100 \\
\hline $\begin{array}{l}\text { Tempo para volta da fertilidade após o uso do } \\
\text { contraceptivo oral combinado }\end{array}$ & 65 & 91,5 & 6 & 8,5 & 71 & 100 \\
\hline $\begin{array}{l}\text { Alteração do leite materno pelas pílulas } \\
\text { exclusivas de progestágeno }\end{array}$ & 63 & 88,7 & 8 & 11,3 & 71 & 100 \\
\hline \multicolumn{7}{|l|}{ Métodos de barreira } \\
\hline Eficácia do preservativo masculino & 34 & 47,9 & 37 & 52,1 & 71 & 100 \\
\hline Uso do preservativo feminino & 63 & 88,7 & 8 & 11,3 & 71 & 100 \\
\hline Uso do diafragma & 28 & 40 & 42 & 60 & 70 & 100 \\
\hline Tempo de ação do espermicida & 51 & 72,9 & 19 & 27,1 & 70 & 100 \\
\hline Durabilidade do DIU & 65 & 97 & 2 & 3 & 67 & 100 \\
\hline \multicolumn{7}{|l|}{ Métodos naturais } \\
\hline Indicação de métodos naturais & 67 & 94,4 & 4 & 5,6 & 71 & 100 \\
\hline Prática do coito interrompido & 71 & 100 & - & - & 71 & 100 \\
\hline Alteração da eficácia do método da Temperatura & 46 & 64,8 & 25 & 35,2 & 71 & 100 \\
\hline Corporal Basal. & & & & & & \\
\hline Prevenção de DST por métodos naturais & 69 & 97,2 & 2 & 2,8 & 71 & 100 \\
\hline $\begin{array}{l}\text { Características do muco cervical durante o } \\
\text { período fértil }\end{array}$ & 34 & 47,9 & 37 & 52,1 & 71 & 100 \\
\hline \multicolumn{7}{|l|}{ Métodos definitivos } \\
\hline Critérios para realizar laqueadura & 48 & 67,6 & 23 & 32,4 & 71 & 100 \\
\hline Relações sexuais após vasectomia & 62 & 87,3 & 9 & 12,7 & 71 & 100 \\
\hline Complicações da vasectomia & 56 & 80 & 14 & 20 & 70 & 100 \\
\hline Abstinência após laqueadura & 31 & 44,3 & 39 & 55,7 & 70 & 100 \\
\hline
\end{tabular}


La mayoría de los profesionales, 58 (81,7\%), acertó cuando respondió como cierto que el fármaco de elección para las mujeres que utilizan anticonceptivos orales por primera vez debe ser con baja dosis. Se sabe que la elección del primer método hormonal a ser utilizado por una mujer siempre debe basarse en una dosis baja de hormonas $(0,03 \mathrm{mg}$ de etinilestradiol o $5 \mathrm{mg}$ de estradiol), debido a una mejor tolerancia y eficacia de estos fármacos. En los casos de sangrado entre las menstruaciones, debe haber una indicación de la píldora de dosis media ${ }^{7,8}$.

Cuando se les preguntó sobre el inicio obligatorio del anticonceptivo oral durante el período menstrual, $40(56,3 \%)$ erraron cuando la respondieron como verdadera. El anticonceptivo oral de preferencia debe ser iniciado desde el primero hasta el quinto día de la menstruación, pero si el anticonceptivo oral se inicia después de este periodo se debe utilizar un método de barrera o la pareja debe abstenerse de tener relaciones sexuales durante siete días, sin la obligación de iniciar el método durante el período menstrual ${ }^{7}$.

Se preguntó a los profesionales si una mujer que amamanta puede utilizar la inyección hormonal cada tres meses. De estos, 29 (40,8\%) se equivocaron al afirmar la cuestión como falsa. La inyección trimestral puede ser utilizada por las mujeres que están amamantando, siempre que cumplan un mínimo de seis semanas después del nacimiento de su hijo ${ }^{5,7}$. Otros métodos son preferibles, pero si estos no están disponibles o no son aceptados, la mujer puede utilizar la inyección anticonceptiva.

Se dijo que se tarda seis meses para el regreso de la fertilidad en una mujer que usa hormonales orales combinados. La mayoría de los enfermeros, 65 (91,5\%) acertaron cuando respondieron a esta pregunta como falsa, ya que el retorno de la fertilidad en mujeres que utilizan la hormona es inmediata y no necesita un tiempo de seis meses ${ }^{8}$.

63 entrevistados $(88,7 \%)$ acertaron cuando respondieron como falsa la cuestión de que las píldoras exclusivas de progestina (PEP) modifican la cantidad o la calidad de la leche materna. Entre las ventajas de las PEP se señala el que no interfiere con la lactancia materna, y no afectan a la calidad o la cantidad de leche ${ }^{7}$.

A los profesionales se les preguntó si los condones pueden ofrecer una eficacia de aproximadamente el $100 \%$ cuando se usa correctamente, $37(52,1 \%)$ erraron el tema cuando lo consideró como falso. El condón tiene una tasa de fracaso del 3\% cuando se utiliza correctamente en todas las relaciones sexuales ${ }^{8}$. Se sabe que el uso del condón es esencial para la prevención de enfermedades de transmisión sexual y gravidez ${ }^{9}$. Su eficiencia es superior al $85 \%{ }^{10}$; y puede llegar a $98 \%$ y $95 \%$ para el condón masculino y femenino respectivamente ${ }^{11}$. El fracaso de los condones se debe a la falta de motivación para parar las caricias antes de la relación y poner el condón. La tasa de fracaso es por lo tanto relacionada con el propio usuario en no usar los condones de manera correcta ${ }^{12}$.

Sobre el condón femenino, a los profesionales se les preguntó si este debe ser evitado durante la menstruación. De éstos, 8 (11,3\%) erraron cuando respondieron que es verdadera. El condón femenino se puede utilizar en todas las relaciones, incluso durante la menstruación, y no es obligatorio no utilizarlo durante el periodo menstrual ${ }^{8}$.

Con relación al diafragma, se le preguntó si debe usarse con espermicida y no retirarlo por 4 horas después del coito. En esta sección, 70 enfermeros respondieron y $42(60 \%)$ se equivocaron al afirmar como verdadero. El espermicida puede mejorar el uso de diafragma ${ }^{5}$. Se recomienda que se utilice una pequeña cantidad de crema o jalea espermicida en el diafragma, pero solo si el espermicida es un método coadjuvante ${ }^{7}$. El diafragma es un 
método eficaz que no causa efectos sistémicos, y el tiempo mínimo adecuado para la retirada después de la relación sexual es de 6 horas y un máximo de 24 horas $^{13}$.

A los profesionales se les preguntó sobre el tiempo de la acción de los espermicidas. De los 70 profesionales que respondieron, 51 (72,9\%) acertaron al decir como verdadero un tiempo de 1 a 2 horas. Se recomienda esperar de 10 a 15 minutos después de insertar el espermicida en forma de tableta o supositorio, sin necesidad de esas cremas, espumas y gelatinas, y su acción es de 1 a 2 horas $^{7}$.

Aunque no sea una realidad de nuestra población, es importante que los profesionales que asisten en el planeamiento familiar tengan conocimiento acerca de este y de todos los otros métodos.

Cuando se les preguntó sobre la duración del DIU, 67 enfermeros respondieron y de éstos, $65(97 \%)$ acertaron a esa pregunta, según la cual su duración es de 5 a 10 años. Esta variación del DIU es por cuenta de su tipo, siendo 5 años para el DIU Multiload Cu-375; de 10 años para el Tcu-380; y de 5 a 7 años, el DIU con levonorgestrel ${ }^{8}$.

Este gran tiempo de duración es una de las razones por las cuales las mujeres eligen este método, además del método dar una mayor autonomía para el usuario ${ }^{14}$.

Se preguntó si los métodos de la tabla, el moco cervical y la temperatura son indicados para las adolescentes y las mujeres durante la menopausia, y $4(5,6 \%)$ enfermeras se equivocaron al decir que esta es verdadera. Se sabe que los métodos de comportamiento están contraindicados para las mujeres con ciclo menstrual irregular ${ }^{7}$, como es el caso de las adolescentes y las mujeres en el período climatérico.

Sobre el ítem que se refería a la posibilidad de existir espermatozoides en el líquido que sale del pene antes de la eyaculación, en la práctica del coito interrumpido, todos los profesionales, 71 (100\%), responden correctamenter como verdadero. El coito interrumpido no se recomienda como el único método anticonceptivo de elección, debido a su alta posibilidad de fracaso a causa de la presencia de espermatozoides en el líquido antes de la eyaculación y la posible falta de control del hombre para interrumpir la relación ${ }^{5}$.

En cuanto al método de la temperatura basal del cuerpo, se dijo que las relaciones durante la noche y la alimentación antes de acostarse pueden alterar la eficacia del método y 25 $(35,2 \%)$ enfermeros se equivocaron al responder que esa pregunta es falsa. Además de estos factores, puede afectar a la eficacia del método de la temperatura basal del cuerpo, el consumo de alcohol, la alteración del sueño, cambios en el medio ambiente y los cambios emocionales ${ }^{8}$.

Con relación a la cuestión que se afirma de que algunas ETS se pueden prevenir por métodos naturales, 69 (97,2\%) enfermeros respondieron que es falsa, acertando, porque no hay ningún método natural de anticoncepción que evite las ETS durante su utilización ${ }^{7}$.

Sobre el método del moco cervical se dijo que este se presenta transparente, elástico, fluido, resbaladizo y en pequeña cantidad durante el período fértil; 37 (52,1\%), enfermeros se equivocaron al responder que esto es cierto, ya que la cantidad es grande y no pequeña ${ }^{7}$. Es importante conocer estas características, principalmente para orientar a las mujeres, que así pueden identificar más fácilmente su período fértil mediante la observación de sólo algunos aspectos de la mucosidad, incluyendo la cantidad, por ser más fácil de reconocer cuando hay cambios. 
Los métodos de comportamiento son los menos utilizados y menos conocidos por la población, que presenta más dificultades en la "utilización" de ellos. Sin embargo, los profesionales deben ser capaces de dar todas las directrices relacionadas con estos métodos a los clientes que buscan atención de salud y están interesados en el uso de un método natural como anticonceptivo.

Una pregunta estaba relacionada con las leyes que rigen la esterilización quirúrgica en Brasil, la Ley 9263 de 1996. Se dijo que es necesario que la mujer tenga dos hijos vivos o que la mujer sea mayor de 25 años. De los encuestados, $23(32,4 \%)$ estaban equivocados diciendo que esta pregunta es falsa.

Algunos autores afirman que las principales causas de error relativo a esta ley están relacionadas con la mala interpretación en la combinación de criterios de edad y número de hijos. Muchos profesionales se suman a los dos criterios, pero solo es necesario uno de ellos, que pueden estar justificadas por la cuestión de que los médicos informan de que las mujeres son jóvenes y hay mucho arrepentimiento. Otro error consiste en aumentar el número mínimo de niños exigidos ${ }^{15}$.

Cuando se le preguntó si el hombre puede tener relaciones sexuales después de una vasectomía sin el riesgo de embarazo, 62 (87,3\%) enfermeros contestan correctamente, pues respondieron que la frase es falsa. Cuando se realiza la vasectomía, es necesario que el hombre realice examen de semen después de 30 eyaculaciones y use el condón u otro método, para que no haya riesgo de embarazo, ya que la primera eyaculación después de la vasectomía puede contener espermatozoides. Por lo tanto, hasta que el examen de semen resulte negativo, el hombre no debe tener relaciones sexuales ${ }^{5,8}$. Este problema es inherente, ya que la vasectomía es un método sencillo y barato (en comparación con la ligadura de trompas), y trae al hombre la responsabilidad conjunta por la anticoncepción.

Acerca de las complicaciones de la vasectomía, se le preguntó si el sangrado puede ser una de ellas. De los 70 profesionales que respondieron, 14 (20\%) enfermeros se equivocaron al decir que esta afirmación es falsa. Muchos profesionales dedican su atención solo al edema y al hematoma como complicaciones de la vasectomía, no conociendo otras posibles complicaciones y no orientando sobre las mismas. Además del edema, pueden ser citados el dolor, el hematoma y el sangrado y la infección local (menos común) como complicaciones de la vascetomia ${ }^{7}$.

Se preguntó cuánto tiempo es necesario que la mujer se abstenga de relaciones sexuales después de realizar la esterilización. Se afirmó que 2 semanas son necesarias. Entre los 70 enfermeros que respondieron, 39 (55,7\%) informaron que la frase es verdadera, lo que es incorrecto. De hecho, después de la cirugía para la esterilización, la mujer debe abstenerse de tener relaciones sexuales durante una semana para garantizar la eficacia del método. La interrupción de las relaciones ocurre si hay aparición de dolor ${ }^{7}$.

Vemos la necesidad de formación de los profesionales para que puedan dedicar mayor cantidad de información a sus clientes y ganar su confianza, estar más cualificados y lograr la eficacia y la continuidad del método anticonceptivo y la autonomía de los clientes, haciendo que ellos también sean responsables y activos en sus propias elecciones y decisiones. 


\section{CONCLUSIÓN}

Los resultados indican la necesidad de formación de enfermeros, con respecto a las prácticas anticonceptivas, con el objetivo de trabajar de manera más activa, añadiendo el conocimiento científico y los valores populares, y dando la oportunidad de aclarar las dudas de los clientes que buscan orientación.

La autonomía del profesional enfermero también depende de su conocimiento, siendo necesario, por tanto, que los enfermeros busquen una actuación independiente y responsable.

También demuestran que los enfermeros deben recibir actualizaciones sobre los métodos anticonceptivos, incluidos los que están disponibles en la unidad de salud, para obtener mejor actuación de enfermería y proporcionar a los clientes informaciones más confiables.

En la búsqueda de garantizar la eficacia del método elegido para la anticoncepción, es necesario que la información proporcionada a los clientes sea correcta y que las mujeres y las parejas no queden con ninguna duda sobre el método elegido. La eficacia de los métodos anticonceptivos depende de la actuación de los profesionales de la salud, incluyendo a la enfermera, pues las informaciones inherentes a los métodos y la forma correcta de pasarlas están directamente relacionadas con la seguridad del uso de los anticonceptivos y la conquista de la autonomía de mujeres y hombres al servicio de la planificación familiar.

\section{REFERENCIAS BIBLIOGRÁFICAS}

1. Lei no 9263, de 12 de janeiro de 1996. 12 Jan 1996. [online] [Acesso em 04 Ago 07]. Disponível em URL: http://www.planalto.gov.br/ccivil/LEIS/L9263.htm

2. Giffin K. Pobreza, desigualdade e equidade em saúde: considerações a partir de uma perspectiva de gênero transversal. Caderno de Saúde Pública 2002; 18 Suppl:103-12.

3. Oliveira F. PAISM, 20 anos depois. In PAISM, 20 anos depois. Encarte: Atenção integral à saúde da mulher. Jornal da rede feminista de saúde 2005; 27: 4-5.

4. Moura ERF, Silva RM. Competência profissional e assistência em anticoncepção. Revista de Saúde Pública 2005; 39(5):795-801.

5. Ministério da Saúde (BR). Direitos Sexuais, direitos reprodutivos e métodos anticoncepcionais. Brasília (DF): Ministério da Saúde; 2006.

6. Budó MLD, Mattioni FC, Machado TS, Ressel LB, Lopes LFD. Quality of life and health promotion throught the perspective of the users of the family healh strategy. Online Brazilian Journal Nursing [online]. 2007; 7(8). Avaliable from: http://www.uff.br/objnursing/index.php/nursing/article/view/j.1676-4285.2008.1104/291

7. Secretaria de Saúde (CE). Saúde reprodutiva e sexual: um manual para a atenção primária e secundária (nível ambulatorial). Fortaleza (CE): Secretaria de saúde; 2002.

8. Ministério da Saúde (BR). Assistência em planejamento familiar: manual técnico. Brasília (DF): Ministério da Saúde; 2002.

9. Falcão Júnior JSP, Rabelo STO, Lopes EM, Freitas LV, Pinheiro AKB, Ximenes LB. Perfil e práticas sexuais de universitários da área da saúde. Esc. Anna Nery Rev Enferm 2007 Mar; 11(1):58-65.

10. Silva CV, Brêtas JRS, Ferreira D, Correa DS, Cintra CC. Uso da camisinha por adolescentes e jovens: avaliação as seqüência dos procedimentos. Acta Paulista de Enfermagem 2004 Out/Dez; 14(4):392-9. 
11. Organización Mundial de la Salud. Critérios médicos de elegibilidad para el uso de anticonceptivos. 3a ed. Ginebra: Organización Mundial de la Salud; 2005.

12. Canella PRB. organizador. Anticoncepção e sexualidade: Um guia para a gravidez. São Paulo: Expressão e arte; 2002.

13. Halckmann S, Lago TG, Barbosa RM, Villela W, Goihman S. O diafragma como método contraceptivo: a experiência de usuárias de serviços públicos de saúde. Cad Saúde Pública 1997 Out/Dez; 13(4):647-57.

14. Moreira MHC, Araújo JNG. Planejamento familiar: autonomia ou encargo feminino? Psicolog. Est. Maringá Set/Dez; 9(3):389-98.

15. Berquó $\mathrm{E}$, Cavenaghi $\mathrm{S}$. Direitos reprodutivos de mulheres e homens face à nova legislação brasileira sobre esterilização voluntária. Cad. Saúde Pública 2003; 19 Suppl. 2:441-53. 